far infra-red regions, we would predict that the analysis will proceed most easily through initial treatment of the far infra-red spectrum followed by detailed analysis of the microwave spectrum.

H. A. Gebbie

N. W. B. STone

Basic Physics Division, National Physical Laboratory,

Teddington, Middlesex.

Research Laboratories,

Polaroid Corporation, Cambridge, Mass.

Spectroscopy Laboratory,

Massachusetts Institute of Technology, Cambridge, Mass.

$1 a$, Bird, G. R., Baird, J. C., et al. (to be published), b, Bird, G. R., J. Chem. Phys., 25, 1040 (1956).'

${ }^{3}$ Bird, G. R., Danti, A., and Lord, R. C., Spectrochimica Acta, 12, 247 (1958).

s Gebbie, H. A., Advances in Quantum Electronics, 155 (Columbia Univ. Press, New York, 1961).

" Curl, R. F., and Kinsey, J. L., J. Chem. Phy8., 35, 1758 (1961).

${ }^{5}$ Lin, C. C., Phys. Rev., 116, 903 (1959).

'Baird, J. C., doctoral thesis, Rice University (1958).

\section{Sedimentation of a Large Number of Particles as a Cluster in Air}

IN an attempt to explain the distribution produced on the ground by the sedimentation of particles from an "instantaneous point source" under the influence of the wind, Davies ${ }^{1}$ assumed that the particles fell with their Stokes velocity and had no influence on the rate of fall of neighbouring particles. The complicating factors of the differing settling velocities, resulting from an extended range of particle sizes, and turbulent diffusion, from the influence of the wind, make it difficult to test this assumption under his experimental conditions. It would, however, be remarkable if particles did not affect each other when falling through air as a cluster, as they do in other fluids ${ }^{2}$.

An examination has been made of the sedimentation of clusters 25-90 cm diameter, composed of small spherical particles of glass and also of 'Diakon', through still air. In each case the particles were exhaustively sieved through sieves which complied with B.S.S. 410 and dispersed as a roughly spherical cluster, the initial distribution along the horizontal axes being Gaussian. An attempt was made to obtain a Gaussian distribution vertically, but this was abandoned when it was found that the behaviour of the cluster was independent of the distribution of the particles for a given size of cloud. Three different types of behaviour have been observed all of which indicate mutual interference in the sedimentation of the particles. In all cases the rate of fall of the cluster exceeded the terminal velocity of the individual particles.

With 'Diakon' particles less than $64 \mu$ diameter, clusters approximately $50 \mathrm{~cm}$ diameter which contained approximately $2 \mathrm{~g}$ of material fell initially as roughly spherical vortices with vertical axes. The circulation of the particles in these clusters was downwards in the centre. This observation was in accord with the behaviour of suspensions in liquids and similar, though of opposite sense, to the rise of masses of hot moist air to form cumulus clouds ${ }^{8}$.

When the particles were larger $(>150 \mu)$ the behaviour of the cluster changed. Immediately on release a circulation of the same nature as above was set up; this was transient and the particles became distributed in the form of a bowl (Fig. 1). These bowl-shaped clusters were formed in all cases when the terminal velocity of the individual particles was greater than about a third of that of the sedimentation rate of the cluster as a whole. It would appear that this distribution of particles resulted from the inability of the individuals to rise at the edges of the vortex at a sufficient velocity to maintain the vortex.

When the weight of material dispersed was increased the cluster fell in a turbulent manner. The mass at which

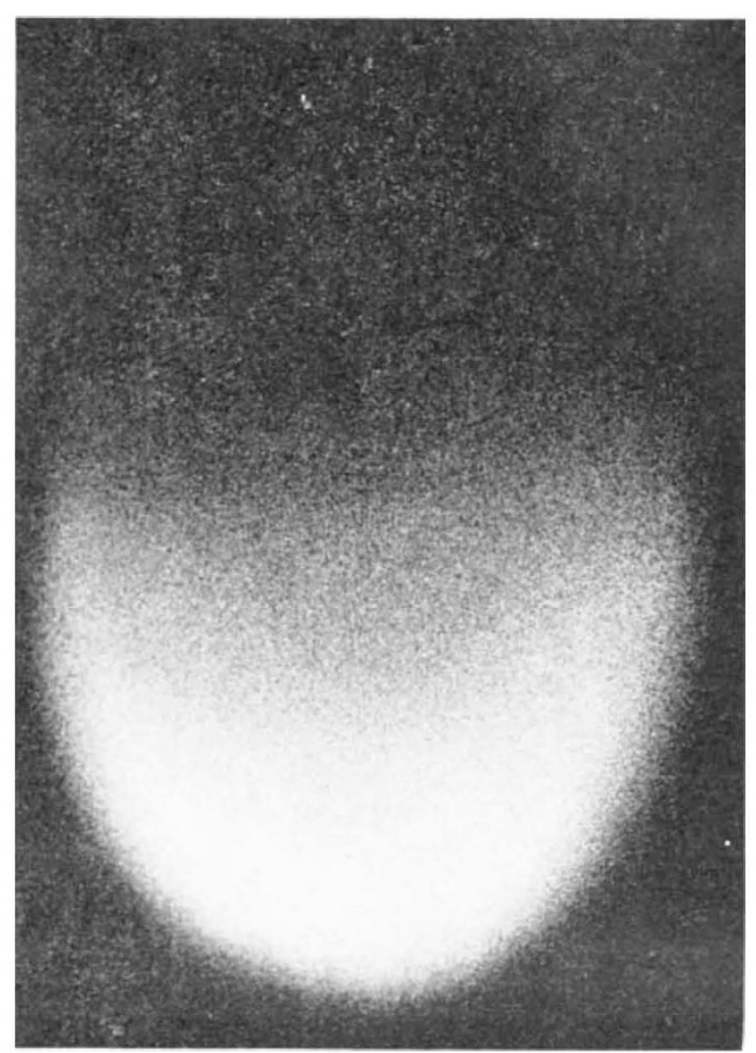

Fig. 1. Bowl-shaped cluster of falling particles. (Courtesy H.M.S.O.)

turbulent behaviour set in increased with increasing particle size and increasing density of the disperse phase.

War Department,

G. W. Srack

Chemical Defence Experimental Establishment, Porton Down,

Salisbury, Wilts.

${ }^{1}$ Davies, D. R., Proc. Camb. Phil. Soc., 46, 506 (1950).

' Slack, G. W., Nature, 200, 466 (1963).

${ }^{3}$ Ludlam, F. H., and Scorer, R. S., Quart. J. Met. Sci., 79, 317 (1953).

\section{GEOPHYSICS}

\section{A Solar Anisotropy in Cosmic Radiation, viewed from a Terrestrial Frame of Reference}

IT is generally recognized that a solar anisotropy in the primary cosmic radiation must be responsible for at least part of the observed terrestrial daily variation of intensity, although it seems that the exact nature of this contribution is not yet clear. However, Rao, McCracken and Venkatesan ${ }^{1}$ have recently presented a method of determ. ining the characteristics of a daily variation of this type. Their method takes into consideration the influence of the Earth's magnetic field and in particular they emphasize the part played by the asymptotic cones of acceptance of cosmic ray detectors. Consequently, using experimental values of the daily variation of neutron intensity averaged over the year 1958, they have been able to postulate a type of anisotropy which appears to account for the complex manner in which the amplitude and phase of the first harmonic of the daily variation depends on the geographical location of the detector. However, it would perhaps be truer to say that they predict that if the Earth's atmosphere and its magnetic field were removed the annual mean diumal variation of intensity observed at the Earth's surface would have the following characteristics: (1) the diurnal variation would be independent 